\title{
TORSION UNITS IN INTEGRAL GROUP RING OF HIGMAN-SIMS SIMPLE GROUP
}

\author{
V.A. BOVDI, A.B. KONOVALOV \\ Dedicated to Professor Béla Csákány on his 75th birthday
}

\begin{abstract}
Using the Luthar-Passi method, we investigate the classical Zassenhaus conjecture for the normalized unit group of the integral group ring of the Higman-Sims simple sporadic group HS. As a consequence, we confirm the Kimmerle's conjecture on prime graphs for this sporadic group.
\end{abstract}

\section{IntRoduction AND MAIN RESUlts}

Let $V(\mathbb{Z} G)$ be the normalized unit group of the integral group ring $\mathbb{Z} G$ of a finite group $G$. One of most interesting conjectures in the theory of integral group ring is the conjecture (ZC) of H. Zassenhaus [25], saying that every torsion unit $u \in V(\mathbb{Z} G)$ is conjugate to an element in $G$ within the rational group algebra $\mathbb{Q} G$.

For finite simple groups, the main tool of the investigation of the Zassenhaus conjecture is the Luthar-Passi method, introduced in 21 to solve the (ZC) for $A_{5}$. Later in [16 M. Hertweck extended and applied it for the investigation of the Zassenhaus conjecture for $\operatorname{PSL}\left(2, p^{n}\right)$. The method proved to be useful for groups containing non-trivial normal subgroups as well. We refer to [5, 17, 15, 16, 17, 18 for recent results. Related results can be found in [1, 22, and [3, 20. In the latter papers weakened versions of the (ZC) were conjectured.

In order to state one of these we introduce some notation. By $\#(G)$ we denote the set of all primes dividing the order of $G$. The Gruenberg-Kegel graph (or the prime graph) of $G$ is the graph $\pi(G)$ with vertices labelled by the primes in \# $(G)$ and there is an edge from $p$ to $q$ if and only if there is an element of order $p q$ in the group $G$. In 20] W. Kimmerle proposed the following:

Conjecture (KC): if $G$ is a finite group then $\pi(G)=\pi(V(\mathbb{Z} G))$.

Obviously, the Zassenhaus conjecture (ZC) implies the Kimmerle conjecture (KC). In 20] it was shown, that (KC) holds for finite Frobenius and solvable groups. We remark that with respect to the so-called $p$-version of the Zassenhaus conjecture the investigation of Frobenius groups was completed by M. Hertweck and the first author in [4. In [7, 8, 9, 11], (KC) was also confirmed for certain Mathieu sporadic simple groups, and in [6] - for some Janko sporadic simple groups.

In this paper we continue these investigations for the Higman-Sims simple sporadic group HS. The main result provides information about the possible torsion units in $V(\mathbb{Z H S})$. An immediate consequence is a positive answer to (KC) for HS.

1991 Mathematics Subject Classification. Primary 16S34, 20C05, secondary 20D08.

Key words and phrases. Zassenhaus conjecture, Kimmerle conjecture, torsion unit, partial augmentation, integral group ring.

The research was supported by OTKA grants No.T 037202, No.T 038059. 
In order to state the result we need to introduce some notation. Let $G$ be a group. Let $\mathcal{C}=\left\{C_{1}, \ldots, C_{n t}, \ldots\right\}$ be the collection of all conjugacy classes of $G$, where the first index denotes the order of the elements of this conjugacy class and $C_{1}=\{1\}$. Suppose $u=\sum \alpha_{g} g \in V(\mathbb{Z} G)$ has finite order $k$. Denote by $\nu_{n t}=\nu_{n t}(u)=\varepsilon_{C_{n t}}(u)=\sum_{g \in C_{n t}} \alpha_{g}$, the partial augmentation of $u$ with respect to $C_{n t}$. From the Berman-Higman Theorem (see [2] and [24], Ch.5, p.102) one knows that $\nu_{1}=\alpha_{1}=0$ and

$$
\sum_{C_{n t} \in \mathcal{C}} \nu_{n t}=1 .
$$

Hence, for any character $\chi$ of $G$, we get that $\chi(u)=\sum \nu_{n t} \chi\left(h_{n t}\right)$, where $h_{n t}$ is a representative of a conjugacy class $C_{n t}$.

The main result is the following.

Theorem 1. Let $G$ denote the Higman-Sims simple sporadic group HS. Let $u$ be a torsion unit of $V(\mathbb{Z} G)$ of order $|u|$. Denote by $\mathfrak{P}(u)$ the tuple

$\left(\nu_{2 a}, \nu_{2 b}, \nu_{3 a}, \nu_{4 a}, \nu_{4 b}, \nu_{4 c}, \nu_{5 a}, \nu_{5 b}, \nu_{5 c}, \nu_{6 a}, \nu_{6 b}, \nu_{7 a}\right.$,

$$
\left.\nu_{8 a}, \nu_{8 b}, \nu_{8 c}, \nu_{10 a}, \nu_{10 b}, \nu_{11 a}, \nu_{11 b}, \nu_{12 a}, \nu_{15 a}, \nu_{20 a}, \nu_{20 b}\right) \in \mathbb{Z}^{23}
$$

of partial augmentations of $u$ in $V(\mathbb{Z} G)$. The following properties hold.

(i) There is no elements of orders 14, 21, 22, 33, 35, 55 and 77 in $V(\mathbb{Z} G)$. Equivalently, if $|u| \notin\{24,30,40,60,120\}$, then $|u|$ coincides with the order of some $g \in G$.

(ii) If $|u| \in\{3,7\}$, then $u$ is rationally conjugate to some $g \in G$.

(iii) If $|u|=2$, the tuple of the partial augmentations of $u$ belongs to the set

$$
\begin{array}{r}
\left\{\mathfrak{P}(u) \mid\left(\nu_{2 a}, \nu_{2 b}\right) \in\{(0,1),(-2,3),(2,-1),(1,0),(3,-2),(-1,2)\},\right. \\
\left.\nu_{k x}=0, k x \notin\{2 a, 2 b\}\right\} .
\end{array}
$$

(iv) If $|u|=5$, the tuple of the partial augmentations of $u$ belongs to the set

$$
\begin{array}{r}
\left\{\mathfrak{P}(u) \mid\left(\nu_{5 a}, \nu_{5 b}, \nu_{5 c}\right) \in\{(-2,-1,4),(-1,-1,3),(0,-1,2),(1,-1,1),\right. \\
(1,3,-3),(0,3,-2),(-3,0,4),(-2,0,3),(1,0,0),(1,4,-4), \\
(-1,0,2),(0,0,1),(0,2,-1),(-1,2,0),(1,2,-2),(0,1,0), \\
(-2,-2,5),(-1,-2,4),(0,-2,3),(1,-2,2),(1,1,-1), \\
\left.(-2,1,2),(-1,1,1)\}, \quad \nu_{k x}=0, k x \notin\{5 a, 5 b, 5 c\}\right\} .
\end{array}
$$

(v) If $|u|=11$, the tuple of the partial augmentations of $u$ belongs to the set

$$
\begin{array}{r}
\left\{\mathfrak{P}(u) \mid\left(\nu_{11 a}, \nu_{11 b}\right)\right) \in\{(5,-4),(4,-3),(-2,3),(2,-1),(-3,4), \\
(-4,5),(1,0),(3,-2),(-1,2),(0,1)\}, \\
\left.\nu_{k x}=0, \quad k x \notin\{11 a, 11 b\}\right\} .
\end{array}
$$

Corollary 1. If $G$ is the Higman-Sims sporadic group, then $\pi(G)=\pi(V(\mathbb{Z} G))$.

\section{Preliminaries}

The following result relates the solution of the Zassenhaus conjecture to vanishing of partial augmentations of torsion units. 
Proposition 1. (see 21] and Theorem 2.5 in [23]) Let $u \in V(\mathbb{Z} G)$ be of order $k$. Then $u$ is conjugate in $\mathbb{Q} G$ to an element $g \in G$ if and only if for each d dividing $k$ there is precisely one conjugacy class $C$ with partial augmentation $\varepsilon_{C}\left(u^{d}\right) \neq 0$.

The next result yield that several partial augmentations are zero.

Proposition 2. (see [15], Proposition 3.1; 16], Proposition 2.2) Let $G$ be a finite group and let $u$ be a torsion unit in $V(\mathbb{Z} G)$. If $x$ is an element of $G$ whose p-part, for some prime $p$, has order strictly greater than the order of the p-part of $u$, then $\varepsilon_{x}(u)=0$.

Another important restriction on the partial augmentations is given by the following result.

Proposition 3. (see [21, 16]) Let either $p=0$ or $p$ is a prime divisor of $|G|$. Suppose that $u \in V(\mathbb{Z} G)$ has finite order $k$ and assume that $k$ and $p$ are coprime when $p \neq 0$. If $z$ is a complex primitive $k$-th root of unity and $\chi$ is either a classical character or a $p$-Brauer character of $G$ then, for every integer $l$, the number

$$
\mu_{l}(u, \chi, p)=\frac{1}{k} \sum_{d \mid k} \operatorname{Tr}_{\mathbb{Q}\left(z^{d}\right) / \mathbb{Q}}\left\{\chi\left(u^{d}\right) z^{-d l}\right\}
$$

is a non-negative integer.

Note that if $p=0$, we will use the notation $\mu_{l}(u, \chi, *)$ for $\mu_{l}(u, \chi, 0)$.

Finally, we shall use the well-known bound for orders of torsion units.

Proposition 4. (see [12]) The order of a torsion element $u \in V(\mathbb{Z} G)$ is a divisor of the exponent of $G$.

\section{Proof of the Theorem}

In this section we denote by $G$ the Higman-Sims simple sporadic group HS. It is well known [13, 14, that

$$
|G|=44352000=2^{9} \cdot 3^{2} \cdot 5^{3} \cdot 7 \cdot 11 \quad \text { and } \quad \exp (G)=9240=2^{3} \cdot 3 \cdot 5 \cdot 7 \cdot 11 .
$$

The character table of $G$, as well as the Brauer character tables (denoted by $\mathfrak{B C T}(p)$, where $p \in\{2,3,5,7,11\}$ ) can be found by the computational algebra system GAP 14, which derives its data from [13, 19. Throughout the paper we will use the notation of GAP Character Table Library for the characters and conjugacy classes of the Higman-Sims group HS.

From the structure of the group HS we know that it possesses elements of orders $2,3,4,5,6,7,8,10,11,12,15$ and 20 . We begin our investigation with units of orders $2,3,5,7$ and 11 . We do no treat the remaining cases $(4,6,8,12,15,20)$, because in these cases the computation is quite complex. Since by Proposition 4 the order of each torsion unit divides the exponent of $G$, it remains to consider in addition only the units of orders 14, 21, 22, 24, 30, 33, 35, 40, 55, 60, 77 and 120. Now we omit five remaining cases: 24, 30, 40, 60 and 120, since these cases are computationally too complicated. We can prove that the order of an unit can not be equal to $14,21,22,33,35,55$ or 77 .

Thus, in this paper we are going to treat the cases when the order of $u$ is: 2,3 , $5,7,11,14,21,22,33,35,55$ and 77 .

- Let $|u| \in\{3,7\}$. Since there is only one conjugacy class in $G$ consisting of elements or order $|u|$, this case follows at once from Proposition 2 . Thus, for units of orders 3 
and 7 we obtained that there is precisely one conjugacy class with non-zero partial augmentation. Proposition 1 then yields part (ii) of the Theorem.

- Let $u$ be an involution. By (11) and Proposition 2 we get $\nu_{2 a}+\nu_{2 b}=1$. Applying Proposition 3 to the character $\chi_{2}$ we get the following system of inequalities

$$
\begin{aligned}
& \mu_{0}\left(u, \chi_{2}, *\right)=\frac{1}{2}\left(6 \nu_{2 a}-2 \nu_{2 b}+22\right) \geq 0 ; \\
& \mu_{1}\left(u, \chi_{2}, *\right)=\frac{1}{2}\left(-6 \nu_{2 a}+2 \nu_{2 b}+22\right) \geq 0 .
\end{aligned}
$$

From the requirement that all $\mu_{i}\left(u, \chi_{j}, p\right)$ must be non-negative integers it can be deduced that $\left(\nu_{2 a}, \nu_{2 b}\right)$ satisfies the conditions of part (iii) of the Theorem.

- Let $u$ be a unit of order 5. By (11) and Proposition 2 we obtain that

$$
\nu_{5 a}+\nu_{5 b}+\nu_{5 c}=1 .
$$

Put $t_{1}=3 \nu_{5 a}-2 \nu_{5 b}-2 \nu_{5 c}$ and $t_{2}=2 \nu_{5 a}-3 \nu_{5 b}+2 \nu_{5 c}$. Again applying Proposition 3 to the characters $\chi_{2}, \chi_{3}$ and $\chi_{4}$, we obtain the system of inequalities

$$
\begin{gathered}
\mu_{0}\left(u, \chi_{2}, *\right)=\frac{1}{5}\left(-4 t_{1}+22\right) \geq 0 ; \quad \mu_{1}\left(u, \chi_{2}, *\right)=\frac{1}{5}\left(t_{1}+22\right) \geq 0 ; \\
\mu_{0}\left(u, \chi_{3}, *\right)=\frac{1}{5}\left(4 t_{2}+77\right) \geq 0 ; \quad \mu_{1}\left(u, \chi_{3}, *\right)=\frac{1}{5}\left(-t_{2}+77\right) \geq 0 ; \\
\mu_{0}\left(u, \chi_{3}, 2\right)=\frac{1}{5}\left(24 \nu_{5 a}-16 \nu_{5 b}+4 \nu_{5 c}+56\right) \geq 0 ; \\
\mu_{0}\left(u, \chi_{4}, 2\right)=\frac{1}{5}\left(28 \nu_{5 a}+8 \nu_{5 b}-12 \nu_{5 c}+132\right) \geq 0 ; \\
\mu_{0}\left(u, \chi_{3}, 3\right)=\frac{1}{5}\left(-4 \nu_{5 a}+16 \nu_{5 b}-4 \nu_{5 c}+49\right) \geq 0,
\end{gathered}
$$

that has only 23 integer solutions $\left(\nu_{5 a}, \nu_{5 b}, \nu_{5 c}\right)$ (they are listed in the part (iv) of the Theorem) such that all $\mu_{i}\left(u, \chi_{j}, p\right)$ are non-negative integers.

- Let $u$ be a unit of order 11. By (11) and Proposition 2 we have

$$
\nu_{2 a}+\nu_{3 a}+\nu_{6 a}=1 .
$$

Applying Proposition 3 to the characters in $\mathfrak{B C T}(3)$ and $\mathfrak{B C T}(5)$ we get the system

$$
\begin{aligned}
\mu_{1}\left(u, \chi_{14}, *\right) & =\frac{1}{11}\left(6 \nu_{11 a}-5 \nu_{11 b}+896\right) \geq 0 ; \\
\mu_{2}\left(u, \chi_{14}, *\right) & =\frac{1}{11}\left(-5 \nu_{11 a}+6 \nu_{11 b}+896\right) \geq 0 ; \\
\mu_{1}\left(u, \chi_{3}, 3\right) & =\frac{1}{11}\left(6 \nu_{11 a}-5 \nu_{11 b}+49\right) \geq 0 ; \\
\mu_{2}\left(u, \chi_{3}, 3\right) & =\frac{1}{11}\left(-5 \nu_{11 a}+6 \nu_{11 b}+49\right) \geq 0 \\
\mu_{1}\left(u, \chi_{9}, 5\right) & =\frac{1}{11}\left(6 \nu_{11 a}-5 \nu_{11 b}+280\right) \geq 0 ; \\
\mu_{2}\left(u, \chi_{9}, 5\right) & =\frac{1}{11}\left(-5 \nu_{11 a}+6 \nu_{11 b}+280\right) \geq 0
\end{aligned}
$$

that has only ten integer solutions $\left(\nu_{11 a}, \nu_{11 b}, \nu_{6 a}\right)$ listed in part (v) of the Theorem.

- Let $u$ be a unit of order 14. By (11) and Proposition 2 we have

$$
\nu_{2 a}+\nu_{2 b}+\nu_{7 a}=1 \text {. }
$$

Put

$$
(\alpha, \beta, \gamma, \delta)=\left\{\begin{array}{lll}
(34,22,90,64), & \text { if } \quad \chi\left(u^{7}\right)=\chi(2 a) ; \\
(26,30,78,76), & \text { if } \quad \chi\left(u^{7}\right)=\chi(2 b) ; \\
(10,3,54,100), & \text { if } \quad \chi\left(u^{7}\right)=-2 \chi(2 a)+3 \chi(2 b) ; \\
(42,14,102,52), & \text { if } \quad \chi\left(u^{7}\right)=2 \chi(2 a)-\chi(2 b) ; \\
(-1,6,114,40), & \text { if } \quad \chi\left(u^{7}\right)=3 \chi(2 a)-2 \chi(2 b) ; \\
(18,38,66,88), & \text { if } \quad \chi\left(u^{7}\right)=-\chi(2 a)+2 \chi(2 b) .
\end{array}\right.
$$


Additionally, we set

$$
t_{1}=6 \nu_{2 a}-2 \nu_{2 b}+\nu_{7 a} \quad \text { and } \quad t_{2}=13 \nu_{2 a}+\nu_{2 b} .
$$

Since $\left|u^{7}\right|=2$, for any character $\chi$ of $G$ we need to consider six cases, defined by part (iii) of the Theorem. Using Proposition 3, it is easy to check, that in all six cases we have the following system of inequalities:

$$
\mu_{0}\left(u, \chi_{3}, *\right)=\frac{1}{14}\left(6 t_{2}+\gamma\right) \geq 0 ; \quad \mu_{7}\left(u, \chi_{3}, *\right)=\frac{1}{14}\left(-6 t_{2}+\delta\right) \geq 0 .
$$

Furthermore, if $\chi\left(u^{7}\right) \in\{\chi(2 a), \chi(2 b), 2 \chi(2 a)-\chi(2 b),-\chi(2 a)+2 \chi(2 b)\}$, then again by Proposition 3 we get the system:

$$
\mu_{0}\left(u, \chi_{2}, *\right)=\frac{1}{14}\left(6 t_{1}+\alpha\right) \geq 0 ; \quad \mu_{7}\left(u, \chi_{2}, *\right)=\frac{1}{14}\left(-6 t_{1}+\beta\right) \geq 0 .
$$

If $\chi\left(u^{7}\right)=-2 \chi(2 a)+3 \chi(2 b)$ then similarly as before, we obtain that

$$
\mu_{0}\left(u, \chi_{2}, *\right)=\frac{1}{14}\left(6 t_{1}+\alpha\right) \geq 0 ; \quad \mu_{2}\left(u, \chi_{2}, *\right)=\frac{1}{14}\left(-t_{1}+\beta\right) \geq 0 .
$$

Finally, if $\quad \chi\left(u^{7}\right)=3 \chi(2 a)-2 \chi(2 b)$ then by Proposition 3 we have

$$
\mu_{1}\left(u, \chi_{2}, *\right)=\frac{1}{14}\left(t_{1}+\alpha\right) \geq 0 ; \quad \mu_{7}\left(u, \chi_{2}, *\right)=\frac{1}{14}\left(-6 t_{1}+\beta\right) \geq 0 .
$$

If we substitute the possible values of $(\alpha, \beta, \gamma, \delta)$ from (3) into (5) - (8), then we can compute the possible values of $t_{1}$ and $t_{2}$ in all of the tree cases. Now we substitute back these values of $t_{1}$ and $t_{2}$ into (4). Then (2) and (4) gives tree linear equations for $\nu_{2 a}, \nu_{2 b}$ and $\nu_{7 a}$. Since $\left|\begin{array}{ccc}1 & 1 & 1 \\ 6 & -2 & 1 \\ 13 & 1 & 0\end{array}\right| \neq 0$, this system has an unique solution, but this solution is not integral.

- Let $u$ be a unit of order 21. By (11) and Proposition 2 we have $\nu_{3 a}+\nu_{7 a}=1$. Using Proposition 3 for the characters $\chi_{2}, \chi_{3}$ of $G$, we get the following system

$$
\begin{aligned}
& \mu_{0}\left(u, \chi_{2}, *\right)=\frac{1}{21}\left(48 \nu_{3 a}+12 \nu_{7 a}+36\right) \geq 0 ; \\
& \mu_{7}\left(u, \chi_{2}, *\right)=\frac{1}{21}\left(-24 \nu_{3 a}-6 \nu_{7 a}+24\right) \geq 0 ; \\
& \mu_{1}\left(u, \chi_{3}, *\right)=\frac{1}{21}\left(5 \nu_{3 a}+72\right) \geq 0,
\end{aligned}
$$

which has no nonnegative integral solution $\left(\nu_{3 a}, \nu_{7 a}\right)$.

- Let $u$ be a unit of order 22. By (1) and Proposition 2 we have

$$
\nu_{2 a}+\nu_{2 b}+\nu_{11 a}+\nu_{11 b}=1
$$

Put

$$
(\alpha, \beta)=\left\{\begin{array}{lll}
(28,16), & \text { if } \quad \chi\left(u^{11}\right)=\chi(2 a) ; \\
(20,24), & \text { if } \quad \chi\left(u^{11}\right)=\chi(2 b) ; \\
(4,4), & \text { if } \quad \chi\left(u^{11}\right)=-2 \chi(2 a)+3 \chi(2 b) ; \\
(36,8), & \text { if } \quad \chi\left(u^{11}\right)=2 \chi(2 a)-\chi(2 b) ; \\
(0,0), & \text { if } \quad \chi\left(u^{11}\right)=3 \chi(2 a)-2 \chi(2 b) ; \\
(12,32), & \text { if } \quad \chi\left(u^{11}\right)=-\chi(2 a)+2 \chi(2 b) .
\end{array}\right.
$$

Moreover set

$$
t_{1}=3 \nu_{2 a}-\nu_{2 b}, \quad t_{2}=13 \nu_{2 a}+\nu_{2 b}, \quad t_{3}=16 \nu_{2 b}+5 \nu_{11 a}-6 \nu_{11 b} .
$$


Since $\left|u^{11}\right|=2$ and $\left|u^{2}\right|=11$, for any character $\chi$ of $G$ we need to consider 60 cases, defined by parts (iii) and (v) of the Theorem. We parameterize these six cases by values of $(\alpha, \beta)$ from (9).

Case 1. Let $(\alpha, \beta)=(28,16)$. Then by Proposition 3 we get the system

$$
\mu_{0}\left(u, \chi_{2}, *\right)=\frac{1}{22}\left(20 t_{1}+\alpha\right) \geq 0 ; \quad \mu_{11}\left(u, \chi_{2}, *\right)=\frac{1}{22}\left(-20 t_{1}+\beta\right) \geq 0,
$$

that has no integral solution.

Case 2. Let $(\alpha, \beta)=(20,24)$. Then by Proposition 3 we get the system

$$
\mu_{0}\left(u, \chi_{2}, *\right)=\frac{1}{22}\left(20 t_{1}+\alpha\right) \geq 0 ; \quad \mu_{11}\left(u, \chi_{2}, *\right)=\frac{1}{22}\left(-20 t_{1}+\beta\right) \geq 0 .
$$

Again by Proposition 3 we obtain that

$$
\begin{gathered}
\mu_{0}\left(u, \chi_{3}, *\right)=\frac{1}{22}\left(10 t_{2}+78\right) \geq 0 ; \quad \mu_{1}\left(u, \chi_{3}, *\right)=\frac{1}{22}\left(t_{2}+76\right) \geq 0 ; \\
\mu_{11}\left(u, \chi_{3}, *\right)=\frac{1}{22}\left(-10 t_{2}+76\right) \geq 0 ; \\
\mu_{1}\left(u, \chi_{14}, *\right)=\frac{1}{22}\left(16 \nu_{2 b}+5 \nu_{11 a}-6 \nu_{11 b}+\gamma\right) \geq 0 ; \\
\mu_{4}\left(u, \chi_{14}, *\right)=\frac{1}{22}\left(-16 \nu_{2 b}-5 \nu_{11 a}+6 \nu_{11 b}+\delta\right) \geq 0,
\end{gathered}
$$

where

$$
(\gamma, \delta)=\left\{\begin{array}{llll}
(886,918), & \text { if } \chi\left(u^{11}\right)=\chi(2 b) & \text { and } & \chi\left(u^{2}\right)=\chi(11 a) ; \\
(875,907), & \text { if } \chi\left(u^{11}\right)=\chi(2 b) & \text { and } & \chi\left(u^{2}\right)=\chi(11 b) ; \\
(930,962), & \text { if } \chi\left(u^{11}\right)=\chi(2 b) & \text { and } & \chi\left(u^{2}\right)=5 \chi(11 a)-4 \chi(11 b) ; \\
(853,885), & \text { if } \chi\left(u^{11}\right)=\chi(2 b) & \text { and } & \chi\left(u^{2}\right)=-2 \chi(11 a)+3 \chi(11 b) ; \\
(897,929), & \text { if } \chi\left(u^{11}\right)=\chi(2 b) & \text { and } & \chi\left(u^{2}\right)=2 \chi(11 a)-\chi(11 b) ; \\
(842,874), & \text { if } \chi\left(u^{11}\right)=\chi(2 b) & \text { and } & \chi\left(u^{2}\right)=-3 \chi(11 a)+4 \chi(11 b) ; \\
(831,863), & \text { if } \chi\left(u^{11}\right)=\chi(2 a) & \text { and } & \chi\left(u^{2}\right)=-4 \chi(11 a)+5 \chi(11 b) ; \\
(908,940), & \text { if } \chi\left(u^{11}\right)=\chi(2 a) & \text { and } & \chi\left(u^{2}\right)=3 \chi(11 a)-2 \chi(11 b) ; \\
(864,896), & \text { if } \chi\left(u^{11}\right)=\chi(2 a) & \text { and } & \chi\left(u^{2}\right)=-\chi(11 a)+2 \chi(11 b) ; \\
(919,951), & \text { if } \chi\left(u^{11}\right)=\chi(2 b) & \text { and } & \chi\left(u^{2}\right)=4 \chi(11 a)-3 \chi(11 b) .
\end{array}\right.
$$

If we substitute the possible values of $(\gamma, \delta)$ into (11) - (12), then it is easy to check that $t_{1}=-1$ and $t_{2}=1$ (also we can calculate $t_{3}$ ). Now we substitute back these values of $t_{1}, t_{2}$ and $t_{3}$ into (10). Then (10) and (10) gives four linear equations for $\nu_{2 a}, \nu_{2 b}, \nu_{11 a}$ and $\nu_{11 b}$. Since $\left|\begin{array}{cccc}1 & 1 & 1 & 1 \\ 3 & -1 & 0 & 0 \\ 13 & 1 & 0 & 0 \\ 0 & 16 & 5 & -6\end{array}\right| \neq 0$, this system has an unique solution, but this solution is not integral.

Case 3. Let $(\alpha, \beta)=(4,4)$. Then by Proposition 3 we get the system

$$
\begin{aligned}
\mu_{0}\left(u, \chi_{2}, *\right) & =\frac{1}{22}\left(20 t_{1}+4\right) \geq 0 ; & \mu_{2}\left(u, \chi_{2}, *\right) & =\frac{1}{22}\left(-2 t_{1}+4\right) \geq 0 ; \\
\mu_{0}\left(u, \chi_{3}, *\right) & =\frac{1}{22}\left(10 t_{2}+54\right) \geq 0 ; & \mu_{11}\left(u, \chi_{3}, *\right) & =\frac{1}{22}\left(-10 t_{2}+100\right) \geq 0 ; \\
\mu_{1}\left(u, \chi_{14}, *\right) & =\frac{1}{22}\left(t_{3}+\gamma\right) \geq 0 ; & \mu_{4}\left(u, \chi_{14}, *\right) & =\frac{1}{22}\left(-t_{3}+\delta\right) \geq 0,
\end{aligned}
$$

where

$$
(\gamma, \delta)=\left\{\begin{array}{lll}
(854,950), & \text { if } & \chi\left(u^{2}\right)=\chi(11 a) ; \\
(843,939), & \text { if } & \chi\left(u^{2}\right)=\chi(11 b) ; \\
(898,994), & \text { if } & \chi\left(u^{2}\right)=5 \chi(11 a)-4 \chi(11 b) ; \\
(821,917), & \text { if } \quad \chi\left(u^{2}\right)=-2 \chi(11 a)+3 \chi(11 b) ; \\
(865,961), & \text { if } \quad \chi\left(u^{2}\right)=2 \chi(11 a)-\chi(11 b) ; \\
(810,906), & \text { if } \quad \chi\left(u^{2}\right)=-3 \chi(11 a)+4 \chi(11 b) ; \\
(799,895), & \text { if } \quad \chi\left(u^{2}\right)=-4 \chi(11 a)+5 \chi(11 b) ; \\
(876,972), & \text { if } & \chi\left(u^{2}\right)=3 \chi(11 a)-2 \chi(11 b) ; \\
(832,928), & \text { if } & \chi\left(u^{2}\right)=-\chi(11 a)+2 \chi(11 b) ; \\
(887,983), & \text { if } & \chi\left(u^{2}\right)=4 \chi(11 a)-3 \chi(11 b) .
\end{array}\right.
$$

By easy calculation we obtain that $t_{1}=2$ and $t_{2} \in\{-1,10\}$. This case is similar to the previous ones, so we can conclude that there is no integral solution. 
Case 4. Let $(\alpha, \beta)=(36,8)$. Then by Proposition 3 we get the system

$$
\mu_{0}\left(u, \chi_{2}, *\right)=\frac{1}{22}\left(20 t_{1}+\alpha\right) \geq 0 ; \quad \mu_{11}\left(u, \chi_{2}, *\right)=\frac{1}{22}\left(-20 t_{1}+\beta\right) \geq 0,
$$

which leads to a contradiction.

Case 5. Let $(\alpha, \beta)=(0,0)$. Then by Proposition 3 we get the system

$$
\begin{array}{rlrl}
\mu_{1}\left(u, \chi_{2}, *\right) & =\frac{1}{22}\left(2 t_{1}\right) \geq 0 ; & & \mu_{11}\left(u, \chi_{2}, *\right)=\frac{1}{22}\left(-20 t_{1}\right) \geq 0 ; \\
\mu_{0}\left(u, \chi_{3}, *\right)=\frac{1}{22}\left(10 t_{2}+114\right) \geq 0 ; & & \mu_{11}\left(u, \chi_{3}, *\right)=\frac{1}{22}\left(-10 t_{2}+40\right) \geq 0 ; \\
\mu_{1}\left(u, \chi_{14}, *\right)=\frac{1}{22}\left(t_{3}+\gamma\right) \geq 0 ; & & \mu_{4}\left(u, \chi_{14}, *\right)=\frac{1}{22}\left(-t_{3}+\delta\right) \geq 0,
\end{array}
$$

where

$$
(\gamma, \delta)=\left\{\begin{array}{lll}
(935,880), & \text { if } & \chi\left(u^{2}\right)=\chi(11 a) \\
(923,859), & \text { if } \quad \chi\left(u^{2}\right)=\chi(11 b) ; \\
(978,914), & \text { if } \quad \chi\left(u^{2}\right)=5 \chi(11 a)-4 \chi(11 b) \\
(901,837), & \text { if } \quad \chi\left(u^{2}\right)=-2 \chi(11 a)+3 \chi(11 b) \\
(945,881), & \text { if } \quad \chi\left(u^{2}\right)=2 \chi(11 a)-\chi(11 b) ; \\
(890,826), & \text { if } \chi\left(u^{2}\right)=-3 \chi(11 a)+4 \chi(11 b) \\
(879,815), & \text { if } \chi\left(u^{2}\right)=-4 \chi(11 a)+5 \chi(11 b) ; \\
(956,892), & \text { if } \chi\left(u^{2}\right)=3 \chi(11 a)-2 \chi(11 b) ; \\
(912,848), & \text { if } \chi\left(u^{2}\right)=-\chi(11 a)+2 \chi(11 b) ; \\
(967,903), & \text { if } \quad \chi\left(u^{2}\right)=4 \chi(11 a)-3 \chi(11 b) .
\end{array}\right.
$$

By easy calculation we obtain that $t_{1}=0$ and $t_{2} \in\{-7,4\}$. This case is similar to the Case 3 , so we can conclude that there is no integral solution in this case too.

Case 6. Finally, let $(\alpha, \beta)=(36,8)$. By Proposition 3 we obtain the system:

$$
\mu_{0}\left(u, \chi_{2}, *\right)=\frac{1}{22}\left(20 t_{1}+12\right) \geq 0 ; \quad \mu_{11}\left(u, \chi_{2}, *\right)=\frac{1}{22}\left(-20 t_{1}+32\right) \geq 0,
$$

which has no integral solution.

- Let $u$ be a unit of order 33. By (11) and Proposition 2 we get $\nu_{3 a}+\nu_{11 a}+\nu_{11 b}=1$. Again, using Proposition 3 we obtain that

$$
\mu_{0}\left(u, \chi_{2}, *\right)=\frac{1}{33}\left(80 \nu_{3 a}+30\right) \geq 0 ; \quad \mu_{11}\left(u, \chi_{2}, *\right)=\frac{1}{33}\left(-40 \nu_{3 a}+18\right) \geq 0,
$$

that has no integral solution.

- Let $u$ be a unit of order 35. By (11) and Proposition 2 we have that

$$
\nu_{5 a}+\nu_{5 b}+\nu_{5 c}+\nu_{7 a}=1 .
$$

Since $\left|u^{7}\right|=5$, for any character $\chi$ we need to consider 23 cases, defined by part (iv) of the Theorem. Using Proposition 3, we divide these 23 cases into five groups:

Group 1. Let $\chi\left(u^{7}\right)$ belongs to the following set

$$
\begin{aligned}
& \{\chi(5 a)-\chi(5 b)+\chi(5 c), \quad \chi(5 a)+4 \chi(5 b)-4 \chi(5 c), \\
& \chi(5 a)+3 \chi(5 b)-3 \chi(5 c), \quad \chi(5 a)-2 \chi(5 b)+2 \chi(5 c), \\
& \chi(5 a)+2 \chi(5 b)-2 \chi(5 c), \quad \chi(5 a)+\chi(5 b)-\chi(5 c), \quad \chi(5 a)\} .
\end{aligned}
$$

Applying Proposition 3 to the character $\chi_{2}$ we construct the following system

$$
\begin{aligned}
& \mu_{5}\left(u, \chi_{2}, *\right)=\frac{1}{35}\left(12 \nu_{5 a}-8 \nu_{5 b}-8 \nu_{5 c}-4 \nu_{7 a}+9\right) \geq 0 ; \\
& \mu_{0}\left(u, \chi_{2}, *\right)=\frac{1}{35}\left(-6\left(12 \nu_{5 a}-8 \nu_{5 b}-8 \nu_{5 c}-4 \nu_{7 a}\right)+16\right) \geq 0,
\end{aligned}
$$

which has no integral solution.

Group 2. Let $\chi\left(u^{7}\right)$ belongs to the following set

$$
\begin{aligned}
\{\chi(5 b), \quad \chi(5 c), \quad-\chi(5 b)+2 \chi(5 c), & 3 \chi(5 b)-2 \chi(5 c), \\
2 \chi(5 b)-\chi(5 c), & -2 \chi(5 b)+3 \chi(5 c)\} .
\end{aligned}
$$


Using Proposition 3 to the character $\chi_{2}$ we get the system

$$
\begin{aligned}
& \mu_{7}\left(u, \chi_{2}, *\right)=\frac{1}{35}\left(18 \nu_{5 a}-12 \nu_{5 b}-12 \nu_{5 c}-6 \nu_{7 a}+26\right) \geq 0 ; \\
& \mu_{0}\left(u, \chi_{2}, *\right)=\frac{1}{35}\left(-72 \nu_{5 a}+48 \nu_{5 b}+48 \nu_{5 c}+24 \nu_{7 a}+36\right) \geq 0,
\end{aligned}
$$

which has no integral solution.

Group 3. Let $\chi\left(u^{7}\right)$ belongs to the following set

$$
\left\{\begin{aligned}
& -2 \chi(5 a)-\chi(5 b)+4 \chi(5 c), \quad-2 \chi(5 b)+3 \chi(5 c), \\
& -2 \chi(5 a)-2 \chi(5 b)+5 \chi(5 c), \quad-2 \chi(5 a)+\chi(5 b)+2 \chi(5 c) \quad\} .
\end{aligned}\right.
$$

By Proposition 3 we have the following system of inequalities

$$
\begin{aligned}
& \mu_{7}\left(u, \chi_{2}, *\right)=\frac{1}{35}\left(18 \nu_{5 a}-12 \nu_{5 b}-12 \nu_{5 c}-6 \nu_{7 a}+16\right) \geq 0 ; \\
& \mu_{0}\left(u, \chi_{2}, *\right)=\frac{1}{35}\left(-72 \nu_{5 a}+48 \nu_{5 b}+48 \nu_{5 c}+24 \nu_{7 a}+76\right) \geq 0,
\end{aligned}
$$

which has no integral solution.

Group 4. Let $\chi\left(u^{7}\right)$ belongs to the following set

$$
\begin{array}{r}
\{-\chi(5 a)-\chi(5 b)+3 \chi(5 c), \quad-\chi(5 a)+2 \chi(5 c), \quad-\chi(5 a)+2 \chi(5 b), \\
-\chi(5 a)-2 \chi(5 b)+4 \chi(5 c), \quad-\chi(5 a)+\chi(5 b)+\chi(5 c)\} .
\end{array}
$$

Using Proposition 3 to the character $\chi_{2}$ we get the system

$$
\begin{aligned}
& \mu_{7}\left(u, \chi_{2}, *\right)=\frac{1}{35}\left(18 \nu_{5 a}-12 \nu_{5 b}-12 \nu_{5 c}-6 \nu_{7 a}+21\right) \geq 0 ; \\
& \mu_{0}\left(u, \chi_{2}, *\right)=\frac{1}{35}\left(-72 \nu_{5 a}+48 \nu_{5 b}+48 \nu_{5 c}+24 \nu_{7 a}+56\right) \geq 0,
\end{aligned}
$$

which has no integral solution.

Group 5. Finally, let $\chi\left(u^{7}\right)=-3 \chi(5 a)+4 \chi(5 c)$. By Proposition 3 we get

$$
\begin{aligned}
& \mu_{0}\left(u, \chi_{3}, *\right)=\frac{1}{35}\left(48 \nu_{5 a}-72 \nu_{5 b}+48 \nu_{5 c}+85\right) \geq 0 ; \\
& \mu_{7}\left(u, \chi_{3}, *\right)=\frac{1}{35}\left(-12 \nu_{5 a}+18 \nu_{5 b}-12 \nu_{5 c}+75\right) \geq 0,
\end{aligned}
$$

which has no integral solution too.

- Let $u$ be a unit of order 55. By (1) and Proposition 2 we have

$$
\nu_{5 a}+\nu_{5 b}+\nu_{5 c}+\nu_{11 a}+\nu_{11 b}=1 \text {. }
$$

Since $\left|u^{11}\right|=5$ and $\left|u^{5}\right|=11$, for any character $\chi$ of $G$ we need to consider 230 cases, defined by parts (iv) and (v) of the Theorem. Using our implementation of the Luthar-Passi method, which we intended to make available in the GAP package LAGUNA [10, we can employ Proposition 3 to construct in all 230 cases the systems of inequalities. Actually in all cases we obtain a system of two inequalities, and a lot of cases lead to the same system. We present here two cases that yield the same system.

Let $\chi\left(u^{11}\right)=\chi(5 a)$ and either $\chi\left(u^{5}\right)=\chi(11 a)$ or $\chi\left(u^{5}\right)=5 \chi(11 a)-4 \chi(11 b)$. By Proposition 3 we obtain the following system of inequalities

$$
\begin{aligned}
& \mu_{5}\left(u, \chi_{2}, *\right)=\frac{1}{55}\left(12 \nu_{5 a}-8 \nu_{5 b}-8 \nu_{5 c}+10\right) \geq 0 \\
& \mu_{0}\left(u, \chi_{2}, *\right)=\frac{1}{55}\left(-10\left(12 \nu_{5 a}-8 \nu_{5 b}-8 \nu_{5 c}\right)+10\right) \geq 0,
\end{aligned}
$$

which has no integral solution such that all $\mu_{i}\left(u, \chi_{j}, p\right)$ are nonnegative integers.

- Let $u$ be a unit of order 77. By (1) and Proposition 2 we obtain that

$$
\nu_{7 a}+\nu_{11 a}+\nu_{11 b}=1 \text {. }
$$


Finally, using Proposition 3 we get the system of inequalities:

$$
\begin{aligned}
& \mu_{0}\left(u, \chi_{2}, *\right)=\frac{1}{77}\left(60 \nu_{7 a}+28\right) \geq 0 \\
& \mu_{0}\left(u, \chi_{3}, 5\right)=\frac{1}{77}\left(-60 \nu_{7 a}+49\right) \geq 0,
\end{aligned}
$$

which has no integral solution such that all $\mu_{i}\left(u, \chi_{j}, p\right)$ are nonnegative integers.

\section{REFERENCES}

[1] V. A. Artamonov and A. A. Bovdi. Integral group rings: groups of invertible elements and classical K-theory. In Algebra. Topology. Geometry, Vol. 27 (Russian), Itogi Nauki i Tekhniki, pages 3-43, 232. Akad. Nauk SSSR Vsesoyuz. Inst. Nauchn. i Tekhn. Inform., Moscow, 1989. Translated in J. Soviet Math. 57 (1991), no. 2, 2931-2958.

[2] S. D. Berman. On the equation $x^{m}=1$ in an integral group ring. Ukrain. Mat. Ž., 7:253-261, 1955.

[3] F.M. Bleher and W. Kimmerle. On the structure of integral group rings of sporadic groups. LMS J. Comput. Math., 3:274-306 (electronic), 2000.

[4] V. Bovdi and M. Hertweck. Zassenhaus conjecture for central extensions of $S_{5}$. J. Group Theory, pages 1-11, to appear, 2007. (E-print arXiv:math.RA/0609435v1).

[5] V. Bovdi, C. Höfert, and W. Kimmerle. On the first Zassenhaus conjecture for integral group rings. Publ. Math. Debrecen, 65(3-4):291-303, 2004.

[6] V. Bovdi, E. Jespers, and A. Konovalov. Torsion units in integral group rings of Janko simple groups. Preprint, pages 1-30, submitted, 2007. (E-print arXiv:math/0608441v3).

[7] V. Bovdi and A. Konovalov. Integral group ring of the first Mathieu simple group. In Groups St. Andrews 2005. Vol. I, volume 339 of London Math. Soc. Lecture Note Ser., pages 237-245. Cambridge Univ. Press, Cambridge, 2007.

[8] V. Bovdi and A. Konovalov. Integral group ring of the Mathieu simple group $M_{23}$. Comm . Algebra, pages 1-9, to appear, 2007. (E-print arXiv:math/0612640v2).

[9] V. Bovdi, A. Konovalov, and S. Linton. Torsion units in integral group ring of the Mathieu simple group $M_{22}$. Preprint, pages 1-12, submitted, 2007. (E-print arXiv:0704.3733v1).

[10] V. Bovdi, A. Konovalov, R. Rossmanith, and Cs. Schneider. LAGUNA - Lie AlGebras and UNits of group Algebras, Version 3.4, 2007. (http://ukrgap.exponenta.ru/laguna.htm).

[11] V. Bovdi, A. Konovalov, and S. Siciliano. Integral group ring of the Mathieu simple group M12. Rend. Circ. Mat. Palermo (2), 56:125-136, 2007.

[12] J.A. Cohn and D. Livingstone. On the structure of group algebras. I. Canad. J. Math., 17:583-593, 1965.

[13] J. H. Conway, R. T. Curtis, S. P. Norton, R. A. Parker, and R. A. Wilson. Atlas of Finite Groups. Oxford University Press, Eynsham, 1985. Maximal subgroups and ordinary characters for simple groups, With computational assistance from J. G. Thackray.

[14] The GAP Group. GAP - Groups, Algorithms, and Programming, Version 4.4.9, 2006. (http://www.gap-system.org).

[15] M. Hertweck. On the torsion units of some integral group rings. Algebra Colloq., 13(2):329$348,2006$.

[16] M. Hertweck. Partial augmentations and Brauer character values of torsion units in group rings. Comm. Algebra, pages 1-16, to appear, 2007. (E-print arXiv:math.RA/0612429v2).

[17] M. Hertweck. Torsion units in integral group rings or certain metabelian groups. Proc. Edinb. Math. Soc., pages 1-22, to appear, 2007.

[18] C. Höfert and W. Kimmerle. On torsion units of integral group rings of groups of small order. In Groups, rings and group rings, volume 248 of Lect. Notes Pure Appl. Math., pages 243-252. Chapman \& Hall/CRC, Boca Raton, FL, 2006.

[19] C. Jansen, K. Lux, R. Parker, and R. Wilson. An Atlas of Brauer Characters, volume 11 of London Mathematical Society Monographs. New Series. The Clarendon Press Oxford University Press, New York, 1995. Appendix 2 by T. Breuer and S. Norton, Oxford Science Publications.

[20] W. Kimmerle. On the prime graph of the unit group of integral group rings of finite groups. In Groups, rings and algebras, volume 420 of Contemporary Mathematics, pages 215-228. AMS, 2006. 
[21] I. S. Luthar and I. B. S. Passi. Zassenhaus conjecture for $A_{5}$. Proc. Indian Acad. Sci. Math. Sci., 99(1):1-5, 1989.

[22] I. S. Luthar and P. Trama. Zassenhaus conjecture for $S_{5}$. Comm. Algebra, 19(8):2353-2362, 1991.

[23] Z. Marciniak, J. Ritter, S. K. Sehgal, and A. Weiss. Torsion units in integral group rings of some metabelian groups. II. J. Number Theory, 25(3):340-352, 1987.

[24] R. Sandling. Graham Higman's thesis "Units in group rings". In Integral representations and applications (Oberwolfach, 1980), volume 882 of Lecture Notes in Math., pages 93-116. Springer, Berlin, 1981.

[25] H. Zassenhaus. On the torsion units of finite group rings. In Studies in mathematics (in honor of A. Almeida Costa) (Portuguese), pages 119-126. Instituto de Alta Cultura, Lisbon, 1974.

V.A. Bovdi

Institute of Mathematics, University of Debrecen

P.O. Box 12, H-4010 Debrecen, Hungary

Institute of Mathematics and Informatics, College of Nyíregyháza

Sóstói Út 31/B, H-4410 Ný́REgYhÁZA, Hungary

E-mail address: vbovdi@math.klte.hu

A.B. Konovalov

School of Computer Science, University of St Andrews,

Jack Cole Building, North Haugh, St Andrews, Fife, KY16 9SX, Scotland

E-mail address: konovalov@member.ams.org 